\title{
Authoring highly generative Interactive Drama
}

\author{
Nicolas Szilas ${ }^{1}$, Olivier Marty ${ }^{2,3}$, Jean-Hugues Réty ${ }^{4}$ \\ ${ }^{1}$ IDtension \\ 1, rue des Trois Couronnes, 75011 Paris, France \\ http://www.idtension.com \\ nicolas.szilas@libertysurf.fr \\ ${ }^{2}$ LSS / ENS-EHESS \\ 48, bd Jourdan, 75014 Paris, France \\ olivier.marty@ens.fr \\ ${ }^{3}$ LATTS / ENPC-UMLV \\ Cité Descartes, 77426 Marne-la-Vallée, France \\ ${ }^{4}$ LINC - Laboratoire Paragraphe /IUT de Montreuil \\ 140, rue de la Nouvelle France, 93100 Montreuil, France \\ jh.rety@iut.univ-paris8.fr
}

\begin{abstract}
.
Authoring non linear narratives is a difficult and challenging issue. In this paper we focus on the process of authoring with the IDtension system, an interactive drama system designed by one of the authors. We report an experiment of realizing a real-size scenario and start from this point to think about nonlinear narratives and the possibilities and limits of the IDtension writing tool. We introduce the linearization/delinearization cycle, as a basic principle of abstract interactive writing.
\end{abstract}

\section{Introduction}

\subsection{Definition of Interactive Drama}

The term "Interactive Drama" (ID) could refer either to existing forms of interactive fiction, video games, participatory theatre, etc. or to futuristic immersive systems, like the Holodeck described in StarTrek. In this paper, we call Interactive Drama an experience on computer such as described in [1]. We propose the following definition of Interactive Drama:

Interactive Drama is a drama on computer where the user is a character. Being a character means being able to perform any action on the fiction world that the other characters can perform.

For instance, if one character gives some information to the user, she should be able to give it to some third character. This definition shows that ID does not exist yet: in existing interactive fictions or story-based video games, simulated characters 
perform actions that the player cannot perform; thus the user is not a character ; rather, she guides a character through a limited set of actions.

\section{$1.2 \quad$ Authoring}

We distinguish three main issues in designing Interactive Dramas:

- How to build ID systems: What narrative principles can constitute the base for such systems? How is it possible to interact with a story? How to overcome the seeming contradiction between story and interactivity? What part takes Artificial Intelligence in this task?

- What is the role of the user: What does it mean for the user to be a character in a story? What is her narrative role in the story? How does she interact with the system? What existing experience is the closest from ID: book, video game, role playing, child playing, etc. [2].

- What is the role of the author: How does the author communicate her intention and values through the ID? Does the author "give up control" in ID? How to "write a story" given the fact that, eventually, the precise course of events in the story is in the hands of the user?

Let us note that these three issues are indeed not independent. We believe the first one to be the most critical. The question of interactive fiction - which is not new has not been solved by writers themselves because it involves sophisticated computing systems. As long as no viable technical solutions exist for ID, one cannot conceive the kind of art or entertainment it could be. This is the reason why our previous publications on the subject $[3,4,5,6]$ concern technical solutions for ID.

A prototype system - called Idtension - now exists. With this system in hand, the next step was to write a convincing story. This step has raised several questions that we discuss in sections 3 with the description of a concrete experience. The IDtension system is presented in section 2 .

Other advanced ID systems describe ID as a combination of elementary scenes or beats $[7,8,9,10,11]$. In opposite, IDtension describes ID with a finer grain, similarly to [12]. With such a fine grain description of drama, authoring becomes even more difficult.

\section{Overview of IDtension}

We provide in this section a general description of the IDtension system. Further details can be found in [6].

\subsection{The simulation of narrative}

Many models of narrative describe a story as a temporal succession of events. Let us mention for instance the Propp model [13], the Heroe's journey or the screen writing handbooks $[14,15,16]$. We found that these models, sometimes called "plot 
models", are not suited to the level of interactivity we target because they are fundamentally linear: the order of events is almost predetermined. On the other hand, the field of ID being historically related to the field of believable agents, the simulation of characters evolving in a virtual world constitutes another basis for ID. However, we have shown in a previous paper [3] that simulating human beings with intelligent agents (as, for instance, in [17]) is not sufficient to generate a well constructed narrative, because fundamentally, narrative contains "inverse causality" [18]. Because of these two opposite approaches of ID, which reflect the traditional opposition between plot-based and character-based drama, there is a tendency to oppose plot-based approaches and simulation-based approaches, as if simulation was to be restricted to the simulation of living or non living matter [19]. IDtension extends simulation to the narrative itself. It is inspired from structuralism, which aimed at describing the narrative as the temporal unfolding of a non-temporal structure [5].

Let us sketch out what are the advantages of this approach over the plot-based approach by considering the reproduction of a visual scene. The plot-based approach is similar to a painting: to each zone in the scene corresponds a point in the painting. Conversely, the simulation-based approach of IDtension is similar to a 3D model: the deep structure of the narrative is modeled, and the story is the result of the simulation of this structure, as well as the deep structure of the simulated object is modeled, and what you see on the screen is the result of the simulation of the $3 \mathrm{D}$ model. The main advantage of simulation is that it facilitates interaction: The user can influence the simulation while the deep structure remains the same.

\subsection{The global architecture}

The IDtension structural model of narrative includes a rule based system which defines the possible actions in the story and how these actions can be chained together. The rules have been inspired from Bremond [20] and Todorov [21]. In addition to these Structuralist models, IDtension includes a user model for sequencing the actions [3,5]. Such user models are also present in other system for ID [7, 22].

The system is composed of the following modules (see Fig. 1):

- The Narrative Logic: this modules calculates the set of actions that are possible at a given stage of the narrative. It is composed of about 40 rules, which are generic for all narratives. Each rule implements a logical condition for an action to be possible. For instance, if a character $A$ knows that a character $B$ has accomplished a task $t$, then $A$ could

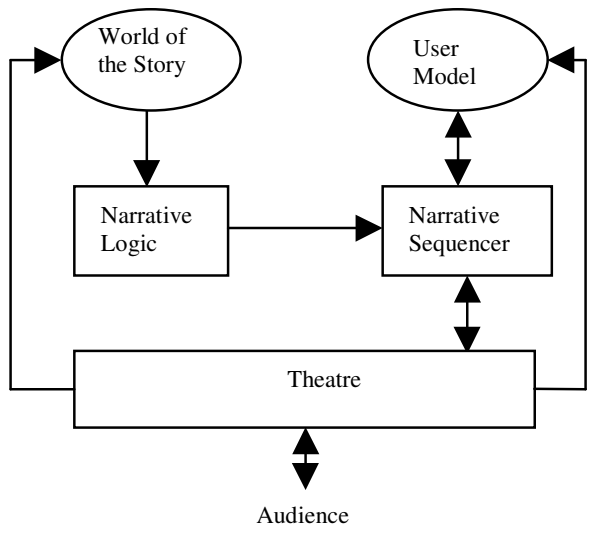

Fig. 1. Architecture of the narrative engine 
congratulate $B$ for having performed $t$. Actions include to inform, to encourage, to dissuade, to accept, to refuse, to perform, to congratulate, to condemn, etc.

- The World of the Story defines the basic entities of the story: characters, objects, places, goals, tasks, sub-tasks or segments, obstacles, states of characters (defined with predicates) and facts concerning the material situation of the world of the story (the fact that a door is closed, for example).

- The User Model stores the history of perceived actions, the perceived values of characters, the opened narrative sequences, etc. This enables to estimate the impact of actions on the user.

- The Narrative Sequencer is the "director" of the system. It receives possible actions from the Narrative Logic, and asks the User Model for the impact of each action, and sends to the theatre the actions to be played.

- The Theatre is responsible for displaying the action(s) and manages the interaction between the computer and the user. Currently, the theatre used in the system is a basic text generator, as output, and a selection among possible actions, as input. Future theatre will typically include a Real-Time 3D interface.

\subsection{The goal/tasks model}

The tasks in a story are parts of the "performative landscape of the story", i.e. what characters can physically do into the story. With IDtension, this includes:

- Goals: states in the world of the story that characters wish to reach.

- Tasks: acts that characters can perform. Performing some tasks enables a character to reach a goal.

- Segments: sub-tasks (task are linear successions of segments)

- Obstacles hinder a segment (thus a task) to be performed. Obstacles play a central role in narrative, in particular in screenwriting (Field Egri) because they allow new developments, suspense, surprise, etc.

There is a subgoaling mechanism based on obstacles. Obstacles can be triggered out depending on conditions. Reaching goals may add or withdraw conditions. Thus, a character can try to reach a goal in order to release an obstacle.

This subgoaling mechanism is to be related to the planing structures used in other ID systems [22,17], although less formalized. Our structure is however more narrative oriented and less Artificial Intelligence oriented.

The IDtension model is not restricted to the performative level. An axiological level - i.e. a level of ethical judgement - is also present with a notion of value. A value is an author-defined axis, like "honesty", "honor", etc. Tasks can be assigned with values, and every character has its own sensibility to values. For instance, some task can be dishonest, so that a character sensible to honesty will be reluctant in performing it. Thus, two tasks can reach the same goal, while being differently evaluated. A task is said to be conflictual to a character if the task can reach one of her goals and if this task is negatively evaluated according to this character's sensibility. 
The task model of IDtension allows for the modeling of various narrative phenomena, like external and internal conflicts, expected and unexpected obstacles, ethical opposition between characters, conflicts of interest between characters, etc.

\subsection{The user model}

Thanks to the richness of the model described above, the Narrative Logic provides a set of actions which are potentially very interesting from a narrative point of view. The actual narrative interest however depends on the proper estimation of the impact on the user. We have identified six criteria to be satisfied in order to have a "good" story:

- motivational consistency: The action is consistent with the goals of the character.

- ethical consistency: The action is consistent with previous actions of the same character, with respect to the axiological system.

- relevance: The action is relevant according to the actions that have just been performed. This criterion corresponds to one of the Grice's maxims.

- cognitive load: The action opens or closes narrative processes, depending on the current number of opened processes. A process is a micro narrative sequence, as defined in [20].

- characterization: The action helps the user understanding the character's features.

- conflict: The action either directly generates some conflict (performing a conflicting task), or the action pushes the user towards a conflicting task (for example by blocking a non-conflicting task, if a conflicting alternative exists).

This set of criteria may be modified in the future. Other interesting criteria have been proposed in [7][23].

\subsection{Results}

A preliminary testing was conducted with a fairly simple story composed of three goals, four tasks and three obstacles ([6]). The test showed the large amount of choices proposed to the user, at each step. However, the example was too simple to show how the story was greatly influenced by user's choices. A new story is now being implemented by the authors of this paper.

\section{Authoring with IDtension}

\subsection{Different levels of authoring}

With the current version of IDtension, the author can write at three levels:

The structures: Complexity of the narrative structure is a very important issue. Every time an author is willing to design a non linear/interactive narrative (this 
happens with ID, hypertext literature or video games), there is some complexity level over which the author looses control over the actual structure of his writing because the graph of this structure becomes much too large. We have found that a particularly relevant level for understanding and writing the story is the "goal/tasks" structure (depicted in Fig. 2). Goal/tasks structures contain only one goal, and several tasks to reach this goal. Here, the complexity is broken down into a number of goal/tasks structures. The link between these goal/tasks structures lies into the notion of obstacle

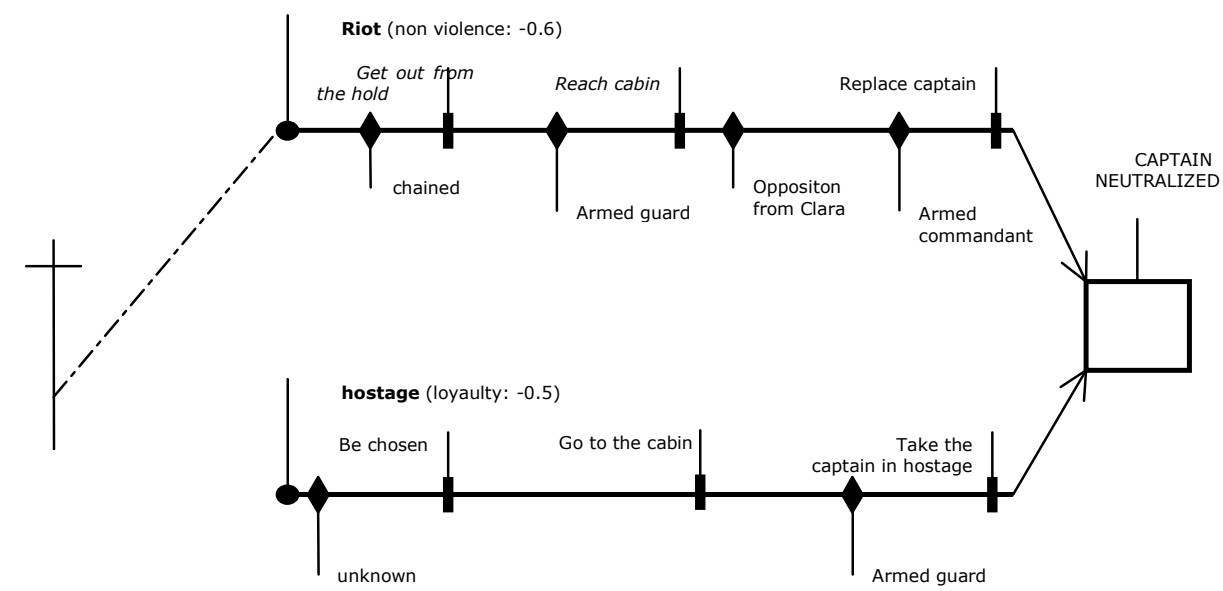

(see previous section). From the author's point of view, obstacles have a meaning in the model of the narrative she is designing out. This understanding helps the author gaining control over the whole structure by considering individual goal/tasks structures, i.e., without drawing out the whole graph of all the goal/tasks structures. For now, the goal/tasks structures are entered by direct coding into the system.

Fig. 2. An example of a goal/tasks structure

The surface text: To each generic action and to each specific entity of a story corresponds a textual form. For example, the action "X INFORM Y that he wants to perform the task t" could be expressed: "[X:name] tells [Y:name]: 'I have decided to [t:infinitive]" ". Parts between brackets are then replaced by the textual forms given by the author for characters or tasks. This is a very basic form of text generation, whose main advantage is that the author has control over all the text. Note that there is no "dialog writing", because with IDtension so far, a dialog is only the result of an exchange of verbal actions. We have chosen a spreadsheet to enter these textual forms, as depicted in Fig. 3, because it is sufficient for our goal and more usable for an author than other formats.

The parameters: There are many parameters in the system which enable to control the narration. For example, one can modify the weighting of each effect, in the calculus of impact of each action. These parameters have the greatest influence on the results. However, it is hard to anticipate the effects of each parameter on the behavior of the story, especially for a non programmer. Thus, in the current stage, the parameters are not chosen by the author himself. They are rather used once the scenario is programmed, during testing. 
In Proc. of the $2^{\text {nd }}$ International Conference on Virtual Storytelling (ICVS 2003), Nov. 2003

\begin{tabular}{|c|c|c|c|c|}
\hline Type & Name & Form & Style & text (original text is in French) \\
\hline \multicolumn{5}{|l|}{ Action } \\
\hline & Inform & & & \\
\hline & & player_is_actor & & \\
\hline & & & neutral & \\
\hline & & & & $\begin{array}{l}\text { You tell [addressee]: "you know, } \\
\text { [content]". }\end{array}$ \\
\hline & & player_is_addressee & & \\
\hline & & & neutral & \\
\hline & & & & [actor] says : "[content]" \\
\hline & & & crude & \\
\hline & & & & [actor] says "hey chap, [content]" \\
\hline & & & polite & \\
\hline & & & & $\begin{array}{l}\text { [actor] tells you :"[addressee:name], } \\
\text { did you know that [content]?" }\end{array}$ \\
\hline & & & & $\begin{array}{l}\text { [actor] says: "do you know that } \\
\text { [content] ?" }\end{array}$ \\
\hline
\end{tabular}

Fig. 3. Extract from the surface text table

\subsection{First experience feedback}

At this point, the IDtension project involved two actors. The computer scientist who built the software and the writer who was in charge of the story. Nicolas Szilas launched IDtension project and built the whole system. He then got in touch with Olivier Marty to help him writing a real size story. The process of imagining and writing the story took several months and presented a few difficulties.

Trying to forget about the techniques of linear novels writing, the author had to imagine a virtual landscape instead of a real story. The conception work was a spatial work instead of a temporal or linear one. The writer had to adapt himself to imagine a wide landscape and a set of actions, letting the control of time to the user of the ID system. Indeed, it is the user of the program who opens her own path in the author's virtual world, who draws her own lines of time within the static landscape.

The author only provides raw material out of which the user builds its own story. It happened that this raw material was shaped by the constraints of the model. A few patterns of writing, such as tasks or obstacles, were added because they matched with the model.

Lastly, it was not intuitive to design the goal/tasks structures from scratch. The writer would often begin by writing or imagining a fragment of story. He would then transform it to make it fit within the goal/tasks structure. 


\subsection{The influence of the tool on authoring}

The author uses structures such as the one shown in Fig. 2 to take an overview of the virtual world he is conceiving. Indeed, the exponential possibilities of the story oblige him to imagine an impressive landscape so huge that it is hardly possible to mentally overlook it without the IDtension device. Therefore the goal/tasks structures are used to write the main lines of the stories, they let details of each sequence to the surface text tables (see Fig. 3).

Instead of the usual word processor, The author had to enter the dialogs into the data base thanks to a spreadsheet. The writing with a spreadsheet looks like the construction of a table more than the writing of a normal text based on a linear oral speech. This leads to a methodic, symmetric and almost fractal way of writing. The author first puts a title to columns and then declines the dialogs lines after lines, obeying to the constraints of each column. This writing methodology typically corresponds to a computer scientist view of literary production, because it splits the text between structure and form, while they are intrinsically interwoven for a literary author [24].

The use of these structures and tables is a new step in the way techniques determine our thoughts ([25]). As a matter of fact, the use of these softwares tends to prevent the author from thinking in a linear way. It obliges his mind to spatial thoughts and symmetric constructions. He is no longer writing a continuous story that would draw a single line between a beginning and an end. He is now forced to imagine a spatial area within which many stories can take place. Therefore, the writing tool he uses determines his thoughts and his production.

\subsection{The linearization / delinearization cycle}

We found it difficult, in our early experience with IDtension, to write at the abstract level. It should be noted that any writing activity implies two levels: an abstract level (the concepts of the story) and a concrete level (the "text" itself). For example, in screenwriting and drama writing, the author usually defines a subject, the characters, the values and conflicts, and then writes the story itself $[15,14]$.

By looking closer at the writing process of non interactive drama, one realizes that the process of abstract writing for those media is not straightforward. For example, it is usually advised to write the backstory of characters, that is what happened to the characters before the beginning of the story, in order to get better characters. In order to construct a representation of characters in their mind, authors start from a story, even if it is a different story from the one that has to be told. Similarly, the drama writer has to "know his characters", to the point where the character "start talking to" her [14 p.41], or to the point where the author and the characters "have chatted about this and that" [16 p.32]. For an author, the most efficient way to build a character is to imagine a situation where he or she is talking to him.

These practices suggest that it is actually not natural to write a story from an abstract perspective only. In non interactive writing, there is a bi-directional process between the abstract description of the story and fragments of stories. The final result is a story. In interactive narrative however, the final result remains abstract. 
Thus, we draw out from our experience a methodological framework of the design of interactive narratives. This framework involves trajectories between three kinds of representations, as represented in Figure 4:

- The abstract representation: it is the abstract description of the story, necessary for making it interactive. In the case of IDtension, it is mainly composed of goal/tasks structures depicted in Fig. 2.

- The "fragments of stories" are produced by the writer (either in her head or with paper and pencil) in order to imagine what could happen during the interaction. It can be linear or a graph of interconnected fragments. this seems to be the natural way of thinking about a story.

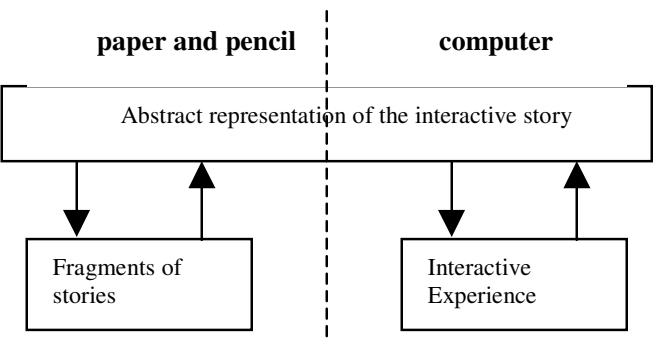

Fig. 4. Four representations involved in the design of an ID. Arrows represent how one representation modifies another representation.

Because IDtension, like

other ID systems, goes beyond linear or multilinear stories, this level of representation is obviously insufficient for describing the story itself.

- The interactive experience: it is the interactive performance that the audience is experiencing while playing with the system.

The flow of information depicted in Fig. 4 can be decomposed into two main design cycles: (1) the conceptual cycle, between the abstract story and the fragments of stories: the author imagine or simulate with paper and pencil the interactive story in order to build the abstract structure; (2) The computer cycle: the abstract structure is coded then executed, which gives feedback to build and modify the abstract structure.

The computer cycle is the most accurate, because the authors can experience the final result of the story. But it is costly, because it involves programming.

\section{Conclusion}

The goal/tasks structures help the author to design non linear narratives. However, there still is a gap between the abstract descriptions of Interactive Drama and the current and natural practices of authors. The linearization/delinearization cycle is the way the author tries to fill up this gap by simulating fragments of story on paper or in her mind. The research of tools that would give to the author a representation of the complexity of non linear narrative structures is crucial but is not likely to eventually provide a complete answer to the problem. We believe that the linearization/delinearization cycle will always exist to some extend. Thus, tools should be designed to assist the flow of information between the different representations of narrative the author is working with. 


\section{References}

1. Bates, J. Virtual Reality, Art, and Entertainment. In Presence: The Journal of Teleoperators and Virtual Environments. Vol. 1. No. 1. MIT Press. (Winter 1992)

2. Ryan, M.-L.: Narrative as Virtual Reality. John Hopkins University Press (2001)

3. Szilas, N.: Interactive Drama on Computer: Beyond Linear Narrative. In Papers from the AAAI Fall Symposium on Narrative Intelligence, Technical Report FS-99-01. AAAI, Press Menlo Park (1999) 150-156. Also http://nicolas.szilas.free.fr/research/aaai99.html

4. Szilas, N.: A New Approach to Interactive Drama: From Intelligent Characters to an Intelligent Virtual Narrator. In Proc. of the Spring Symposium on Artificial Intelligence and Interactive Entertainment (Stanford CA, March 2001), AAAI Press, 72-76.

5. Szilas, N.: Structural Models for Interactive Drama. In the proceedings of the $2^{\text {nd }}$ International Conference on Computational Semiotics for Games and New Media (Augsburg, Germany, Sept. 2002)

6. Szilas, N.: IDtension: a narrative engine for Interactive Drama. In Göbel et al. (eds) Proc. TIDSE'03. Frauenhofer IRB Verlag, (2003)

7. Weyhrauch, P. Guiding Interactive Drama. Ph.D. Dissertation, Tech report CMUCS-97109, Carnegie Mellon University (1997)

8. Stern A., Matteas M.: Integrating Plot, Character and Natural Language Processing in the Interactive Drama Façade. In Göbel et al. (eds) Proc. TIDSE'03. Frauenhofer IRB Verlag, (2003)

9. Donikian, S. HPTS: a Behaviour Modelling Language for Autonomous Agents. In : Fifth International Conference on Autonomous Agents, ACM Press, Montreal, Canada (2001)

10. Machado, I., Paiva, A., and Brna, P. Real characters in virtual stories - Promoting interactive story-creation activities. In Proceedings of the First International Conference on Virtual Storytelling (ICVS 2001). Lecture Notes in Computer Science 2197, Springer Verlag (2001) 127-134

11. Majerko, B., John Laird. Building an Interactive Drama Architecture. . In Göbel et al. (eds) Proc. TIDSE'03. Frauenhofer IRB Verlag (2003)

12. Sgouros, N. M.: Dynamic, User-Centered Resolution in Interactive Stories. In Pollack, M. (ed.) IJCAI'97 Proceedings of the 15th International Joint Conference on Artificial Intelligence. Morgan Kaufmann Publishers, San Francisco (1997)

13. Propp, V.: Morphologie du conte. Seuil, Paris (1928/1970)

14. Field S.: Screenplay - The Foundations of Screenwriting. $3^{\text {rd }}$ edn. : Dell Publishing, NewYork (1984)

15. Jenn, P.: Techniques du scénario. FEMIS, Paris (1991)

16. Egri, L.: The Art of Dramatic Writing. Simon \& Schuster (1946)

17. Cavazza, M., Charles, F., Mead, S. J.: Characters in Search of an author: AI-based Virtual Storytelling. In Proceedings of the First International Conference on Virtual Storytelling (ICVS 2001). Lecture Notes in Computer Science 2197, Springer Verlag (2001) 145-154

18. Genette, G: Figures II. Seuil, Paris (1969)

19. Frasca, G. Simulation versus Narrative: Introduction to Ludology. in Mark et al. (Eds.) Video/Game/Theory. Routledge, (2003, in press)

20. Bremond, C.: Logique du récit. Seuil, Paris (1974)

21. Todorov, T. Les transformations narratives. Poétiques, 3 (1970) 322-333

22. Riedl, O., Micheal Young. Character-focused Narrative. Subm. to Virtual Reality (2003)

23. Bailey, P. Searching for Storiness: Story-Generation from a Reader's Perspective. In Proc. AAAI Fall Symposium on Narrative Intelligence (North Falmouth MA, 1999), AAAI Press

24. Davis, M., Michael Travers. A Brief Overview of the Narrative Intelligence Reading Group. In Papers from the AAAI Fall Symposium on Narrative Intelligence, Technical Report FS-99-01. AAAI, Press Menlo Park (1999) 11-16

25. Goody, J. La raison graphique, Editions de Minuit, Le sens commun (1995) 